\title{
Solving Generally Constrained Generalized Linear Variational Inequalities Using the General Projection Neural Networks
}

\author{
Xiaolin Hu, Student Member, IEEE, and Jun Wang, Fellow, IEEE
}

\begin{abstract}
Generalized linear variational inequality (GLVI) is an extension of the canonical linear variational inequality. In recent years, a recurrent neural network $(\mathrm{NN})$ called general projection neural network (GPNN) was developed for solving GLVIs with simple bound (often box-type or sphere-type) constraints. The aim of this paper is twofold. First, some further stability results of the GPNN are presented. Second, the GPNN is extended for solving GLVIs with general linear equality and inequality constraints. A new design methodology for the GPNN is then proposed. Furthermore, in view of different types of constraints, approaches for reducing the number of neurons of the GPNN are discussed, which results in two specific GPNNs. Moreover, some distinct properties of the resulting GPNNs are also explored based on their particular structures. Numerical simulation results are provided to validate the results.
\end{abstract}

Index Terms-Generalized linear variational inequality (GLVI), recurrent neural networks (NNs), global asymptotic stability, global exponential stability, optimization.

\section{INTRODUCTION}

$\mathbf{L}$ INEAR variational inequality (LVI) is to find $x^{*} \in \mathcal{X}$ such that

$$
\left(M x^{*}+p\right)^{T}\left(x-x^{*}\right) \geqslant 0 \quad \forall x \in \mathcal{X}
$$

where $M \in R^{n \times n}, p \in R^{n}$, and $\mathcal{X} \subset R^{n}$ is a closed convex set. LVI and nonlinear variational inequality (NVI) (which is obtained by replacing $M x+p$ in (1) with a nonlinear vector-valued function $F(x)$ mapping from $R^{n}$ to $R^{n}$ ) have numerous applications such as economic equilibrium modeling, traffic network equilibrium modeling, and structural analysis [1], [2]. Numerous numerical algorithms have been proposed for solving LVIs and NVIs (e.g., [3]-[6]).

During the past two decades, several recurrent neural networks (NNs) were developed for solving LVIs and NVIs, as well as related optimization problems (e.g., see [7]-[12] and the references therein). On one hand, these NNs serve as new computational models for solving these problems in real time. On the

Manuscript received July 29, 2006; revised December 21, 2006; accepted March 25, 2007. This work was supported by the Research Grants Council of the Hong Kong Special Administrative Region, China, under Project CUHK4165/ 03E.

The authors are with the Department of Mechanical and Automation Engineering, The Chinese University of Hong Kong, Shatin, New Territories, Hong Kong (e-mail: jwang@mae.cuhkedu.hk).

Color versions of one or more of the figures in this paper are available online at http://ieeexplore.ieee.org.

Digital Object Identifier 10.1109/TNN.2007.899753 other hand, the dynamic systems governing the NNs may shed lights to the development of new numerical algorithms.

Specifically, when $\mathcal{X}$ is a box set, i.e., $\mathcal{X}=\left\{x \in R^{n} \mid l_{i} \leqslant\right.$ $\left.x_{i} \leqslant u_{i}, \forall i=1, \ldots, n\right\}$, the following projection NN model was developed for solving LVIs and has been extensively studied in the literature (e.g., [7], [8], [13], and [14])

$$
\frac{d x}{d t}=\lambda W\left\{-x+P_{\mathcal{X}}((I-\alpha M) x-\alpha p)\right\}
$$

where $\lambda \in R, W \in R^{n \times n}$, and $\alpha \in R$ are positive constants, and $P_{\mathcal{X}}(x)=\left(P_{\mathcal{X}}\left(x_{1}\right), \ldots, P_{\mathcal{X}}\left(x_{n}\right)\right)^{T}$ with

$$
P_{\mathcal{X}}\left(x_{i}\right)=\left\{\begin{array}{ll}
l_{i}, & x_{i}<l_{i} \\
x_{i}, & l_{i} \leqslant x_{i} \leqslant u_{i} . \\
u_{i}, & x_{i}>u_{i}
\end{array} .\right.
$$

An extension of LVI (1) is as follows: Find $x^{*} \in R^{n}$ such that $N x^{*}+q \in \mathcal{X}$ and

$$
\left(M x^{*}+p\right)^{T}\left(x-N x^{*}-q\right) \geqslant 0 \quad \forall x \in \mathcal{X}
$$

where $M, N \in R^{n \times n} ; p, q \in R^{n}$; and $\mathcal{X}$ is a closed convex set in $R^{n}$. For convenience, this problem is termed the generalized linear variational inequality (GLVI) hereafter. It has many scientific and engineering applications as well, one of which refers to the extended linear-quadratic programming, as discussed in [15].

Based on the projection NN model (2), the following NN model was developed for solving GLVIs when $\mathcal{X}$ is a box set [16], [17]:

$$
\frac{d x}{d t}=\lambda W\left\{-N x+P_{\mathcal{X}}((N-M) x+q-p)-q\right\} .
$$

However, in applications, $\mathcal{X}$ is often a polyhedron defined by a set of linear equalities and inequalities. Other than some refined NN models for solving generally constrained LVIs (e.g., see [10]), no NN has been proposed for solving generally constrained GLVIs. In this paper, we present some new design methods to develop NNs for solving such GLVIs. Toward this goal, we focus on the following NN, which differs slightly from (5) with an additional parameter $\alpha \in R$ :

$$
\frac{d x}{d t}=\lambda W\left\{-N x+P_{\mathcal{X}}((N-\alpha M) x+q-\alpha p)-q\right\}
$$

where $\alpha>0$. According to Lemmas 2 and 3 in Section II, the equilibrium points of (6) coincide with those of (5). However, the additional parameter $\alpha$ in (6) will play an important role in establishing several further stability results of the $\mathrm{NN}$ (see Section III). Though the previous NN arises initially for solving 
GLVIs with box-type constraints, it will be seen in Section IV that its variants can solve GLVIs with general polyhedral constraints; so it is important to explore its properties fully, which will be the main topic of Section III. For convenience, in what follows, we call the NN in (6) the general projection neural network or simply GPNN. In Section IV, we are not satisfied by just designing a universal NN for solving GLVIs with any constraints. Actually, after such an NN is formulated, much of our effort will be devoted to reduce the number of neurons in the NNs for solving specific GLVIs subject to various constraints. Section V provides several illustrative examples and a comparative study with two iterative algorithms. Finally, Section VI concludes the paper.

\section{PRELIMINARIES}

Throughout the paper, the following notations are used. The transpose of a real matrix $A$ is denoted by $A^{T}$. The symmetric part of a square matrix $A$ is denoted by $A^{S}$, which means $(A+$ $A^{T}$ )/2. A square matrix $A$ is said to be positive definite (positive semidefinite), denoted by $A>0(A \geqslant 0)$, if $x^{T} A x>0$ $\left(x^{T} A x \geqslant 0\right) \forall x \neq 0$. Similarly, $A$ is said to be negative definite (negative semidefinite), denoted by $A<0(A \leqslant 0)$, if $-A>0$ $(-A \geqslant 0) . \theta_{\min }(A)$ and $\theta_{\max }(A)$ denote the minimum and the maximum eigenvalues of a real symmetric matrix $A$, respectively. $\|x\|=\sqrt{\sum_{i=1}^{n} x_{i}^{2}}$ denotes the $L_{2}$ norm of a vector $x$. $\|A\|=\sqrt{\theta_{\max }\left(A^{T} A\right)}$ denotes the $L_{2}$ norm of a square matrix $A$. $I$ denotes an identity matrix. $\mathcal{X}^{*}$ stands for the solution set of GLVI (4), which is assumed to be nonempty, and $\mathcal{X}^{e}$ stands for the equilibrium set of GPNN (6).

Definition 1: A real square matrix $A$ is said to be Lyapunov diagonally stable (Lyapunov diagonally semistable), denoted by $A \in \mathcal{L} \mathcal{D} \mathcal{S}(A \in \mathcal{L} \mathcal{D S S})$, if there exists a diagonal matrix $\Gamma>0$ such that $\Gamma A>0(\Gamma A \geqslant 0)$.

Definition 2: A real square matrix $A$ is said to be additively diagonally stable, denoted by $A \in \mathcal{A D S}$, if $\alpha I+A \in \mathcal{L D} \mathcal{S}$ for any $\alpha>0$.

Many practical conditions can be found in [8], [14], and [18] to test the Lyapunov diagonal stability of a matrix. If a matrix is $\mathcal{L D S S}$, then it is necessary to be $\mathcal{A D S}$ [19].

In the following, let $\mathcal{G} \mathcal{L}$ denote the class of globally Lipschitz continuous and monotone nondecreasing functions, i.e., those $g$ satisfying that there exists $\bar{l}_{i}$ such that $\forall \xi, \rho \in R$ and $\xi \neq \rho$

$$
0 \leqslant \frac{g_{i}(\xi)-g_{i}(\rho)}{\xi-\rho} \leqslant \bar{l}_{i}, \quad i=1, \ldots, n .
$$

From (3), it is easy to verify the following fact.

Lemma 1: $P_{\mathcal{X}}(x) \in \mathcal{G} \mathcal{L}$ with $\bar{l}_{i}=1, \forall i=1, \ldots, n$.

Lemma 2 [20]: $\mathcal{X}^{a}=\mathcal{X}^{*}$, where $\mathcal{X}^{a}$ is the solution set of the following:

$$
P_{\mathcal{X}}((N-M) x+q-p)=N x+q
$$

Lemma 3: $\mathcal{X}^{b}=\mathcal{X}^{*}$, where $\mathcal{X}^{b}$ is the solution set of the following:

$$
P_{\mathcal{X}}((N-\alpha M) x+q-\alpha p)=N x+q \quad \forall \alpha \in R, \alpha>0 .
$$

Proof: The proof is straightforward by using Lemma 2, thus omitted.

From Lemma 3, when $W$ is nonsingular, the equilibrium set of GPNN (6) $\mathcal{X}^{e}$ coincides with $\mathcal{X}^{*}$.

Lemma 4 [21, Th. 1.12]: Let $A$ be a Hermitian matrix partitioned as

$$
A=\left(\begin{array}{ll}
A_{11} & A_{12} \\
A_{12}^{H} & A_{22}
\end{array}\right)
$$

where $A_{12}^{H}$ denotes the conjugate transpose of $A_{12}$. Then, the following hold:

1) $A>0$ if and only if both $A_{11}>0$ and $A / A_{11}>0$;

2) $A \geqslant 0$ if and only if both $A_{11}>0$ and $A / A_{11} \geqslant 0$; where $A / A_{11}=A_{22}-A_{12}^{H} A_{11}^{-1} A_{12}$ is called the Schur complement of $A_{11}$ in $A$.

\section{STABILITY RESULTS}

In this section, we present some existing and new stability results of GPNN (6) for solving GLVIs with box-type constraint as described in (3).

The following lemma follows from [16], [17, Corollaries 3 and 4], and Lemma 3 directly.

\section{Lemma 5:}

1) Let $W=I$ in (6). If $N+\alpha M$ is symmetric and positive definite, and $M^{T} N \geqslant 0$, then GPNN (6) is stable in the sense of Lyapunov and globally convergent to a solution of (4). In particular, if the solution is unique, GPNN (6) is globally asymptotically stable. Furthermore, if $M^{T} N>0$, then GPNN (6) is globally exponentially stable.

2) Let $W=(N+\alpha M)^{T}$ in (6). If $M^{T} N \geqslant 0$, then $\mathcal{X}^{*}=$ $\mathcal{X}^{e}$, and GPNN (6) is stable in the sense of Lyapunov and globally convergent to an equilibrium point. In particular, if the solution is unique, GPNN (6) is globally asymptotically stable. Furthermore, if $M^{T} N>0$, then GPNN (6) is globally exponentially stable.

It is seen from Lemma 5 that GPNN (6) with $W=(N+$ $\alpha M)^{T}$ seems to have better theoretical properties than with $W=I$. However, the NN with $W=I$ has its own usefulness. On one side, $W=I$ results in fewer connections of the $\mathrm{NN}$ in circuits implementation than $W=(N+\alpha M)^{T}$. On the other side, if we study the properties of GPNN (6) from other viewpoints rather than relying on positive definiteness or positive semidefiniteness of $M^{T} N$ as Lemma 5 does, many different stability results can be obtained. First, one of the salient advantages for $W=I$ is that it is the simplest condition that ensures $\mathcal{X}^{*}=\mathcal{X}^{e}$ (see Lemma 3); so, in the following, our theoretical analysis of GPNN (6) is stated by letting $W=I$.

By assumption, $\mathcal{X}^{*} \neq \emptyset$. Let $x^{*} \in \mathcal{X}^{*}$. By means of the coordinate translation $z=x-x^{*}$, GPNN (6) with $W=I$ can be put into the equivalent form

$$
\frac{d z}{d t}=\lambda(-N z+g(U z))
$$

where $U=N-\alpha M, g(U z)=\left(g_{1}\left(u_{1} z\right), \ldots, g_{n}\left(u_{n} z\right)\right)^{T}$, $g_{i}\left(u_{i} z\right)=P_{\mathcal{X}}\left(u_{i}\left(z+x^{*}\right)+q_{i}-\alpha p_{i}\right)-P_{\mathcal{X}}\left(u_{i} x^{*}+q_{i}-\right.$ $\left.\alpha p_{i}\right)$, and $u_{i} \in R^{1 \times n}$ denotes the $i$ th row of $U$. Let $L(z)=$ $\operatorname{diag}\left(l_{1}(z), l_{2}(z), \ldots, l_{n}(z)\right)$, where $l_{i}(z)$ satisfies $g_{i}\left(u_{i} z\right)=$ 
$l_{i}(z) u_{i} z i=1, \ldots, n$. According to Lemma $1, l_{i}(z) \in[0,1]$. Then, (7) can be rewritten as

$$
\frac{d z}{d t}=\lambda(-N+L(z) U) z
$$

Based on (7) and (8), two equivalent forms of GPNN (6), the following two theorems are derived.

Theorem 1: If there exists a matrix $P>0$ such that for any $i=1, \ldots, n$

$$
P\left(-N+n U_{i}\right)+\left(-N+n U_{i}\right)^{T} P \leqslant 0
$$

and for at least one $i \in\{1, \ldots, n\}$ the strict previous inequality holds, where $U_{i}=U E_{i}, U=N-\alpha M$, and $E_{i}$ is an $n \times n$ matrix with zero elements except for an element of one in its $i$ th row and $i$ th column, then GPNN (6) with $W=I$ is globally convergent to a solution of (4). In particular, if (4) has a unique solution, GPNN (6) is globally asymptotically stable.

Proof: Define a Lyapunov function $V(z(t))=z^{T} P z$ and compute the time derivative of $V(z(t))$ along the positive half trajectory of (8). Similarly to the analysis of [14, Th. 3], we can derive $d V(z(t)) / d t \leqslant 0$ and the equality holds if and only if $z=0$, which shows that the system (8) is stable in the sense of Lyapunov. Since $P>0$, all level sets of $V$ are bounded. It follows that for any initial point $z\left(t_{0}\right)=x\left(t_{0}\right)-x^{*} \in R^{n}$, there exists a convergent subsequence $\left\{z\left(t_{k}\right)\right\}$ such that

$$
\lim _{k \rightarrow \infty} z\left(t_{k}\right)=\hat{z}
$$

where $\hat{z}=\hat{x}-x^{*}$ and $\hat{x} \in \mathcal{X}^{*}$. Define a Lyapunov function again

$$
\hat{V}(z(t))=(z-\hat{z})^{T} P(z-\hat{z})=(x-\hat{x})^{T} P(x-\hat{x}) .
$$

It is easy to see that $\hat{V}(z)$ decreases along the trajectory of (8) and satisfies $\hat{V}(\hat{z})=0$. Therefore, for any $\varepsilon>0$, there exists $q>0$ such that, for all $t \geqslant t_{q}$

$$
\theta_{\min }\left(P^{S}\right)\|z(t)-\hat{z}\|^{2} \leqslant \hat{V}(z(t)) \leqslant \hat{V}\left(z\left(t_{q}\right)\right)<\varepsilon
$$

where $\theta_{\min }\left(P^{S}\right)>0$. Therefore, $\lim _{t \rightarrow \infty} z(t)=\hat{z}$ and $\lim _{t \rightarrow \infty} x(t)=\hat{x}$. It follows that GPNN (6) is globally convergent to a solution of (4). In particular, if (4) has a unique solution, GPNN (6) is globally asymptotically stable.

Theorem 2: GPNN (6) with $W=I$ is globally exponentially stable at the unique solution of (4) if there exists a matrix $P>0$ such that either of the following holds:

1) $Q_{1}=P N+N^{T} P>0$ and $\left\|P+P^{T}\right\|\|N-\alpha M\|<$ $\theta_{\min }\left(Q_{1}^{S}\right)$

2) $Q_{2}=P M+M^{T} P>0$ and $2\|P\|\|N-\alpha M\|<$ $\alpha \theta_{\min }\left(Q_{2}^{S}\right)$.

Proof:

1) First, in view of Lemma 1 , we have $\|g(U z)\| \leqslant\|U\|\|z\|$, where $U=N-\alpha M$. Consider the Lyapunov function $V(z(t))=z^{T} P z$. As $P>0, \theta_{\min }\left(P^{S}\right) \cdot\|z\|^{2} \leqslant V(z) \leqslant$ $\theta_{\max }\left(P^{S}\right) \cdot\|z\|^{2}$, where $\theta_{\max }\left(P^{S}\right) \geqslant \theta_{\min }\left(P^{S}\right)>0$.
Computing the time derivative of $V(z)$ along the trajectory of (7) yields

$$
\begin{aligned}
\frac{d V(z(t))}{d t} & =z^{T}\left(P+P^{T}\right) \frac{d z}{d t} \\
& =\lambda\left\{-z^{T}\left(P N+N^{T} P\right) z+z^{T}\left(P+P^{T}\right) g(U z)\right\} \\
& \leqslant \lambda\left\{-\theta_{\min }\left(Q_{1}^{S}\right)+\left\|P+P^{T}\right\|\|U\|\right\}\|z\|^{2} \\
& \leqslant-\beta V(z(t))
\end{aligned}
$$

where

$$
\beta=\frac{\lambda\left(\theta_{\min }\left(Q_{1}^{S}\right)-\left\|P+P^{T}\right\|\|U\|\right)}{\theta_{\max }\left(P^{S}\right)}>0 .
$$

It follows

$V(z(t)) \leqslant V\left(z\left(t_{0}\right)\right) \exp \left(-\beta\left(t-t_{0}\right)\right)$

and

$$
\|z(t)\| \leqslant \sqrt{V\left(t_{0}\right) / \theta_{\min }\left(P^{S}\right)} \exp \left(-\beta\left(t-t_{0}\right) / 2\right) \quad \forall t \geqslant t_{0} .
$$

Then, GPNN (6) is globally exponentially stable at $x^{*}$.

2) Consider the same Lyapunov function $V(z(t))$ as defined in part 1), and compute the time derivative of $V(z)$ along the positive half trajectory of (8)

$$
\begin{aligned}
& \frac{d V(z(t))}{d t} \\
&=z^{T}\left(P+P^{T}\right) \frac{d z}{d t} \\
&=\lambda z^{T}\left\{P(-N+L(z) U)+(-N+L(z) U)^{T} P\right\} z \\
&=\lambda z^{T}\left\{P(-N+U)+(-N+U)^{T} P\right. \\
&\left.\quad-P(I-L(z)) U-U^{T}(I-L(z)) P\right\} z \\
&=\lambda z^{T}\left\{-\alpha Q_{2}-P(I-L(z)) U-U^{T}(I-L(z)) P\right\} z \\
& \leqslant \lambda\left\{-\alpha z^{T} Q_{2} z+2\|P\|\|I-L(z)\|\|U\|\|z\|^{2}\right\} \\
& \leqslant \lambda\left\{-\alpha \theta_{\min }\left(Q_{2}^{S}\right)+2\|P\|\|U\|\right\}\|z\|^{2} \\
& \leqslant-\beta V(z(t))
\end{aligned}
$$

where

$$
\beta=\frac{\lambda\left(\alpha \theta_{\min }\left(Q_{2}^{S}\right)-2\|P\|\|U\|\right)}{\theta_{\max }\left(P^{S}\right)}>0 .
$$

In the previous reasoning, we use the fact $I-L(z)=\operatorname{diag}(1-$ $\left.l_{i}(z)\right)$ and $\|I-L(z)\|=\max \left(1-l_{i}(z)\right) \leqslant 1$. Similarly as in part 1) we can conclude that GPNN (6) is globally exponentially stable at $x^{*}$.

If the positive-definite matrix $P$ in Theorem 2 is required to be diagonal, the conditions $Q_{1}>0$ and $Q_{2}>0$ become $Q_{1} \in \mathcal{L D S}$ and $Q_{2} \in \mathcal{L D S}$, respectively. If, furthermore, $P$ is assumed to be an identity matrix, we immediately have Corollary 1.

Corollary 1: GPNN (6) with $W=I$ is globally exponentially stable at the unique solution of (4) if any of the following holds:
1) $N>0,\|N-\alpha M\|<\theta_{\min }\left(N^{S}\right)$;
2) $M>0,\|N-\alpha M\|<\alpha \theta_{\min }\left(M^{S}\right)$; 
3) $N=I, M>0, \alpha<\theta_{\min }\left(M+M^{T}\right) /\|M\|^{2}$;

4) $M=I, N>0, \alpha>\|N\|^{2} / \theta_{\min }\left(N+N^{T}\right)$.

Proof: Parts 1) and 2) follow from Theorem 2 by setting $P=I$ in the theorem. Parts 3) and 4) are special cases of parts 1) and 2), respectively. We only prove part 4) because part 3) can be reasoned similarly. In view of $\|N\|^{2}=\theta_{\max }\left(N^{T} N\right)$, we have

$$
\alpha>\frac{\theta_{\max }\left(N^{T} N\right)\|y\|^{2}}{\theta_{\min }\left(N+N^{T}\right)\|y\|^{2}} \geqslant \frac{y^{T} N^{T} N y}{y^{T}\left(N+N^{T}\right) y}
$$

for any $y \in R^{n}$ satisfying $\|y\|=1$, which follows:

$$
y^{T} N^{T} N y-\alpha y^{T}\left(N+N^{T}\right) y<0
$$

and

$$
y^{T}\left(N^{T} N-\alpha\left(N+N^{T}\right)+\alpha^{2} I\right) y<\alpha^{2}\left\|y^{2}\right\| .
$$

This is equivalent to

$$
\|(N-\alpha I) y\|^{2}<\alpha^{2} .
$$

Then

$$
\|N-\alpha I\|=\max _{\|y\|=1}\|(N-\alpha I) y\|<\alpha .
$$

From part 2) of the corollary, part 4) follows.

It is observed that some conditions in Lemma 5 and Theorem 2 implicitly require the nonsingularity of $N$. In fact, if this condition holds, we can let $W=N^{-1}$ in (6) and obtain some additional results about GPNN (6). Actually, in this case, by using a variable transformation $y=N x+q$, we can rewrite GPNN (6) equivalently as

$$
\left.\frac{d y}{d t}=\lambda\left\{-y+P_{\mathcal{X}}\left(\left(I-\alpha M N^{-1}\right) y+\alpha M N^{-1} q-\alpha p\right)\right)\right\} .
$$

The first observation on the previous system is that it shares a similar structure with GPNN (6) in the following sense: The two matrices $N$ and $M$ in GPNN (6) now become $I$ and $M N^{-1}$, respectively. Corollary 2 then follows from part 1) or part 3) of Corollary 1.

Corollary 2: Assume that $N$ is nonsingular and there exits $\alpha>0$ such that $\left\|I-\alpha M N^{-1}\right\|<1$, then GPNN (6) with $W=N^{-1}$ and such a choice of $\alpha$ is globally exponentially stable at the unique solution of (4).

Remark 1: If $M^{T} N>0$, there always exists $\alpha>0$ that fulfills the requirements in Corollary 2. In fact, one can choose $\alpha<$ $\theta_{\min }\left(M N^{-1}+N^{-T} M^{T}\right) /\left\|M N^{-1}\right\|^{2}$ to ensure the global exponential convergence of the NN. However, in view of Lemma 5 , if the network complexity is not a critical concern in hardware implementation, it would be better to choose $W=\left(N+\alpha M^{T}\right)$ since, in this case, there is no other restrictions on $\alpha$ if only it is positive; otherwise, we can choose $W=I$ in GPNN (6) with an appropriate $\alpha$.

Remark 2: Similar to Corollary 2 which follows from part 1) of Corollary 1, some other conditions from part 2) of Corollary
1 can be derived to ensure the global exponential stability of GPNN (6). However, definitely those conditions will include $M N^{-1}>0$, which indicates that the corresponding results have little meaning by considering Lemma 5 and Remark 1.

The second observation on system (9) is that it is a special case of the system studied in [14], and thus most stability results in [14] can be applied here to obtain specific results for (9). One such elegant result is presented in Theorem 3.

Theorem 3: Assume that $N$ is nonsingular and $M N^{-1} \in$ $\mathcal{L D S}$, then GPNN (6) with $W=N^{-1}$ is globally exponentially stable at the unique solution of (4).

Proof: In view of Lemma 1, in [14, Th. 6], by setting $\bar{L}=$ $I, D=I, W=I-\alpha M N^{-1}$, we obtain the desired result.

If furthermore $N-\alpha M$ is nonsingular, by using another variable transformation $z=\left(I-\alpha M N^{-1}\right) y$, we can rewrite (9) as

$$
\left.\frac{d z}{d t}=\lambda\left\{-z+\left(I-\alpha M N^{-1}\right) P \mathcal{X}\left(z+\alpha M N^{-1} q-\alpha p\right)\right)\right\} .
$$

Note that the previous system falls into a very general class of NNs that has been extensively studied in the NN community in the last two decades (e.g., see [8], [18], [19], [22], and many references therein). Hence, these existing stability results can be utilized to state the stability of GPNN (6). For example, Theorem 4 reflects some new advancements in this regard.

Theorem 4: Assume that $N$ is nonsingular and there exits $\alpha>0$ such that $N-\alpha M$ is nonsingular. Consider GPNN (6) with $W=N^{-1}$.

1) If $\alpha M N^{-1}-I \in \mathcal{A D S}$, then the $\mathrm{NN}$ is globally convergent to a solution of the GLVI. In particular, if (4) has only one solution, then the $\mathrm{NN}$ is globally asymptotically stable.

2) If there exist positive numbers $\varrho_{1}, \varrho_{2}, \ldots, \varrho_{n}$ such that $\sum_{j=1}^{n} \varrho_{j}\left|c_{i j}\right|<\varrho_{i}, \forall i=1, \ldots, n$, where $\left\{c_{i j}\right\}=I-$ $\alpha M N^{-1}$, then the $\mathrm{NN}$ is globally exponentially stable at the unique solution of (4).

Proof: Part 1) follows from [19, Th. 2] and a similar analysis in proving Theorem 1; and part 2) follows from [22, Th. 1].

We emphasize that the stability results about the systems in the form of (9) and (10) are actually abundant in the literature. Only a few of them, which are general and easily testable, are considered in Theorems 3 and 4. Interested readers are suggested to consult the references mentioned previously to derive more stability results for GPNN (6).

Finally, we give a remark on the effect of the scaling factor $\lambda$ of GPNN (6).

Remark 3: From the proofs of the stability results presented in this section, it can be concluded that the larger $\lambda$ in (6) is, the faster the NN converges. The fact that an NN converges to a point does not imply that the $\mathrm{NN}$ will absolutely reach that point. But if the stability results are proved by using the Lyapunov methods, it is usually safe to conclude that the convergence time of GPNN (6) is finite if $\lambda$ is large enough. Actually, except the second part of Theorem 4 which is proved by employing the comparison method [22], all the stability results about GPNN (6) presented in this section are proved by using the Lyapunov methods. Moreover, it is easily seen that the Lyapunov functions 
constructed in the proofs, denote by $V(x(t))$, always satisfy the following:

1) $V(x(t)) \geqslant 0, \forall t \geqslant t_{0}$ and $V(x(t))=0$ if and only if $x(t)=x^{*}$, where $x^{*} \in \mathcal{X}^{*}$;

2) $d V(x(t)) / d t \leqslant-\lambda \phi(x(t))$, where $\phi(x(t))$ is a continuous function satisfying $\phi(x(t))>0 \forall t \geqslant t_{0}$ and $\phi(x(t))=0$ if and only if $x(t)=x^{*}$.

Now, consider the case that the initial point $x\left(t_{0}\right)$ is not an equilibrium point of (6). Then, $\phi\left(x\left(t_{0}\right)\right)>0$ and $\phi(x(t)) \geqslant 0$. Since $\phi(x(t))$ is continuous, there exists $\tau>0, \delta>0$ such that $\phi(x(t)) \geqslant \delta, \forall t \in\left[t_{0}, t_{0}+\tau\right)$. By considering $d V(x(t)) / d t \leqslant$ $-\lambda \phi(x(t)), \forall t>t_{0}+\tau$, we have

$$
\begin{aligned}
V(x(t)) & \leqslant V\left(x\left(t_{0}\right)\right)-\lambda \int_{t_{0}}^{t} \phi(x(\xi)) d \xi \\
& \leqslant V\left(x\left(t_{0}\right)\right)-\lambda \delta \tau .
\end{aligned}
$$

Note that $V(x(t))=0$ if and only if $x(t)=x^{*}$. Suppose $x(t)$ will never reach $x^{*}$ for $t \geqslant t_{0}$, then the last term of the previous equation should be greater than zero, which implies $\lambda<$ $V\left(x\left(t_{0}\right)\right) /(\delta \tau)$. On the contrary, if we take $\lambda \geqslant V\left(x\left(t_{0}\right)\right) /(\delta \tau)$, $x(t)$ will reach $x^{*}$ in finite time. Therefore, in order to accelerate the convergence rate of GPNN (6), it would be a good strategy to make the scaling factor $\lambda$ as large as possible in model implementation.

\section{Generally CONSTRAINED GLVIS}

In this section, we consider the GLVI with $\mathcal{X}$ defined as

$$
\mathcal{X}=\left\{x \in R^{n} \mid x \in \Omega_{x}, A x \in \Omega_{y}, B x=c\right\}
$$

where $A \in R^{h \times n}, B \in R^{r \times n}, c \in R^{r}$, and $\Omega_{x}, \Omega_{y}$ being box sets defined by $\Omega_{x}=\left\{x \in R^{n} \mid \underline{x} \leqslant x \leqslant \bar{x}\right\}$ and $\Omega_{y}=\{y \in$ $\left.R^{h} \mid \underline{y} \leqslant y \leqslant \bar{y}\right\}$ with $\underline{x}, \bar{x}, \underline{y}$, and $\bar{y}$ being constant vectors. Without loss of generality, any component of $\underline{x}$ and $y$ can be $-\infty$, and any component of $\bar{x}$ and $\bar{y}$ can be $\infty$. For convenience, in (11), $x \in \Omega_{x}$ are termed bound constraints, $A x \in \Omega_{y}$ termed inequality constraints, and $B x=c$ termed equality constraints hereafter. Though the bound and equality constraints can be unified into inequality constraints (see Section IV-A), we distinguish them because they can be handled with different techniques which may lead to more efficient computational schemes.

\section{A. GLVIs With General Constraints}

We first consider the general case of $\mathcal{X}$ when all constraints are present in (11). Define another box set

$$
\tilde{\Omega}_{y}=\left\{y \in R^{h+r} \mid\left(\underline{y}^{T}, c^{T}\right)^{T} \leqslant y \leqslant\left(\bar{y}^{T}, c^{T}\right)^{T}\right\} .
$$

Then, (11) becomes

$$
\mathcal{X}=\left\{x \in R^{n} \mid x \in \Omega_{x}, \tilde{A} x \in \tilde{\Omega}_{y}\right\}
$$

where $\tilde{A}=\left(A^{T}, B^{T}\right)^{T}$. The following proposition establishes a necessary and sufficient condition for a solution to the GLVI.
Proposition 1: $x^{*} \in R^{n}$ is a solution of the GLVI with $\mathcal{X}$ defined by (11) if and only if there exists $y^{*} \in R^{h+r}$ such that $N x^{*}+q \in \Omega_{x}, \tilde{A} N x^{*}+\tilde{A} q \in \tilde{\Omega}_{y}$, and

$$
\begin{cases}\left(M x^{*}+p-\tilde{A}^{T} y^{*}\right)^{T}\left(x-N x^{*}-q\right) \geqslant 0 & \forall x \in \Omega_{x} \\ \left(y^{*}\right)^{T}\left(y-\tilde{A} N x^{*}-\tilde{A} q\right) \geqslant 0 & \forall y \in \tilde{\Omega}_{y} .\end{cases}
$$

Proof: Observe that $N x^{*}+q$ solves the following linear programming problem:

$$
\begin{aligned}
& \min \Xi(x)=\left(M x^{*}+p\right)^{T}\left(x-N x^{*}-q\right) \\
& \text { s.t. } x \in \mathcal{X}
\end{aligned}
$$

where $\mathcal{X}$ is a polyhedron defined by (12). By noticing that $\tilde{A} x \in$ $\tilde{\Omega}_{y}$ is equivalent to

$$
\tilde{A} x-\xi=0, \quad \xi \in \tilde{\Omega}_{y}
$$

where $\xi \in R^{h+r}$ is a new variable introduced to the previous linear programming problem, we define the Lagrangian function on $\Omega_{x} \times \widetilde{\Omega}_{y} \times R^{h+r}$ for the problem

$$
L(x, \xi, y)=\Xi(x)-y^{T}(\tilde{A} x-\xi) .
$$

According to the saddle point theorem [23], $N x^{*}+q \in \mathcal{X}$ is a minimum of (14) if and only if $x^{*} \in \Omega_{x}$ and there exist $\xi^{*} \in \tilde{\Omega}_{y}, y^{*} \in R^{h+r}$ such that $\left(N x^{*}+q, \xi^{*}, y^{*}\right)$ is a saddle point of $L$; i.e.,

$$
\begin{array}{r}
L\left(N x^{*}+q, \xi^{*}, y\right) \leqslant L\left(N x^{*}+q, \xi^{*}, y^{*}\right) \leqslant L\left(x, \xi, y^{*}\right) \\
\forall x \in \Omega_{x}, \quad \xi \in \tilde{\Omega}_{y} ; \quad y \in R^{h+r} .
\end{array}
$$

Define a function $\phi(y)=-L\left(N x^{*}+q, \xi^{*}, y\right)$ on $R^{h+r}$, which is clearly a linear function. The left inequality in (15) implies that

$$
\phi\left(y^{*}\right)=\min \phi(y) \quad \forall y \in R^{h+r}
$$

which is equivalent to $\nabla_{y} \phi\left(y^{*}\right)=0$, i.e.,

$$
\tilde{A} N x^{*}+\tilde{A} q-\xi^{*}=0 .
$$

Because $\xi^{*} \in \tilde{\Omega}_{y}$, we have $\tilde{A} N x^{*}+\tilde{A} q \in \tilde{\Omega}_{y}$. Define another function $\varphi(x, \xi)=L\left(x, \xi, y^{*}\right)$ on $\Omega_{x} \times \tilde{\Omega}_{y}$, which is also a linear function. The right inequality in (15) implies that

$$
\varphi\left(N x^{*}+q, \xi^{*}\right)=\min \varphi(x, \xi) \quad \forall x \in \Omega_{x}, \quad \xi \in \tilde{\Omega}_{y}
$$

which is equivalent to the following variational inequalities [24]:

$$
\begin{cases}\nabla_{x}^{T} \varphi\left(N x^{*}+q, \xi^{*}\right)\left(x-N x^{*}-q\right) \geqslant 0 & \forall x \in \Omega_{x} \\ \nabla_{\xi}^{T} \varphi\left(N x^{*}+q, \xi^{*}\right)\left(\xi-\xi^{*}\right) \geqslant 0 & \forall \xi \in \tilde{\Omega}_{y}\end{cases}
$$

i.e.,

$$
\begin{cases}\left(M x^{*}+p-\tilde{A}^{T} y^{*}\right)^{T}\left(x-N x^{*}-q\right) \geqslant 0 & \forall x \in \Omega_{x} \\ \left(y^{*}\right)^{T}\left(\xi-\xi^{*}\right) \geqslant 0 & \forall \xi \in \tilde{\Omega}_{y} .\end{cases}
$$


Substituting $\xi^{*}=\tilde{A} N x^{*}+\tilde{A} q$ into previous equation and replacing $\xi$ with $y$ result in (13), which establishes the proposition.

Let

$$
\begin{aligned}
\tilde{M} & =\left(\begin{array}{cc}
M & -\tilde{A}^{T} \\
0 & I
\end{array}\right) \quad \tilde{p}=\left(\begin{array}{c}
p \\
0
\end{array}\right) \quad \tilde{N}=\left(\begin{array}{cc}
N & 0 \\
\tilde{A} N & 0
\end{array}\right) \\
\tilde{q} & =\left(\begin{array}{c}
q \\
\tilde{A} q
\end{array}\right)
\end{aligned}
$$

and $\tilde{U}=\Omega_{x} \times \tilde{\Omega}_{y}$, then (13) can be written in the following compact form:

$$
\left(\tilde{M} u^{*}+\tilde{p}\right)^{T}\left(u-\tilde{N} u^{*}-\tilde{q}\right) \geqslant 0 \quad \forall u \in \tilde{U}
$$

where $u=\left(x^{T}, y^{T}\right)^{T}$. The conditions in Proposition 1 are equivalent to finding $u^{*} \in R^{n+h+r}$ satisfying $\tilde{N} x^{*}+\tilde{q} \in \tilde{U}$ and (16). It is to say, the original GLVI with a general polyhedral underlying set has been converted to another GLVI with a box underlying set $\tilde{U}$, which can be solved by using the following specific GPNN:

$$
\frac{d u}{d t}=\lambda W\left\{-\tilde{N} u+P_{\tilde{U}}((\tilde{N}-\alpha \tilde{M}) u+\tilde{q}-\alpha \tilde{p})-\tilde{q}\right\}
$$

where $\lambda>0$ and $\alpha>0$ are two scalars, $W \in R^{(n+h+r) \times(n+h+r)}$, and $P_{\tilde{U}}(\cdot)$ is defined similarly as $P_{\mathcal{X}}(\cdot)$ in (3). The output of the NN is simply $x(t)$, the first part of the state $u(t)$. For the convenience of later discussion, let $\tilde{U}^{e}=\Omega_{x}^{e} \times \Omega_{y}^{e}$ denote the set of equilibrium points of (17).

As NN (17) is a special case of GPNN (6), all the stability results of the GPNN presented in Section III can be applied here. In fact, all results there with $N, M, p, q$, and $\mathcal{X}$ replaced by $\tilde{N}, \tilde{M}, \tilde{p}, \tilde{q}$, and $\tilde{U}$ can be used here to state the properties of (17). Clearly, if we do so, it is, in general, inevitable that these stability results will involve requirements on the constraint parameters such as $A, B$, and $c$ in (11) besides $N$ and $M$. But there is at least one exception. See Theorem 5.

Theorem 5: Consider GPNN (17) with $W=(\tilde{N}+\alpha \tilde{M})^{T}$ for solving the GLVI with $\mathcal{X}$ defined in (11). The following hold.

1) If $M^{T} N \geqslant 0$, then $\mathcal{X}^{*}=\Omega_{x}^{e}$, and the state trajectory of the $\mathrm{NN}$ is stable in the sense of Lyapunov and globally convergent to an equilibrium point in $\tilde{U}^{e}$.

2) If, furthermore, $M^{T} N>0$, then the output trajectory $x(t)$ of the $\mathrm{NN}$ is globally asymptotically stable at the unique solution $x^{*}$ of the problem.

Proof: Part 1) is directly from Proposition 1 and Lemma 5 by noting that

$$
\tilde{M}^{T} \tilde{N}=\left(\begin{array}{cc}
M^{T} N & 0 \\
0 & 0
\end{array}\right) \geqslant 0
$$

because $M^{T} N \geqslant 0$ by assumption. We are now concerned with part 2). To prove the global asymptotical stability of the output trajectory $x(t)$, according to Lemma 5 it is only needed to show that the solution of (4) is unique if $M^{T} N>0$. Suppose that $\mathcal{X}^{*}$ has two distinct points $x^{\prime}$ and $x^{\prime \prime}$. Then, correspondingly,
(16) has two distinct solutions $u^{\prime}=\left(\left(x^{\prime}\right)^{T},\left(y^{\prime}\right)^{T}\right)^{T}$ and $u^{\prime \prime}=$ $\left(\left(x^{\prime \prime}\right)^{T},\left(y^{\prime \prime}\right)^{T}\right)^{T}$ according to Proposition 1. Therefore

$$
\begin{array}{ll}
\left(\tilde{M} u^{\prime}+\tilde{p}\right)^{T}\left(u-\tilde{N} u^{\prime}-\tilde{q}\right) \geqslant 0 & \forall u \in \tilde{U} \\
\left(\tilde{M} u^{\prime \prime}+\tilde{p}\right)^{T}\left(u-\tilde{N} u^{\prime \prime}-\tilde{q}\right) \geqslant 0 & \forall u \in \tilde{U} .
\end{array}
$$

Substituting $\tilde{N} u^{\prime \prime}+\tilde{q}$ for $u$ in the first inequality above, and $\tilde{N} u^{\prime}+\tilde{q}$ for $u$ in the second inequality yields

$$
\begin{aligned}
& \left(\tilde{M} u^{\prime}+\tilde{p}\right)^{T}\left(\tilde{N} u^{\prime \prime}-\tilde{N} u^{\prime}\right) \geqslant 0 \\
& \left(\tilde{M} u^{\prime \prime}+\tilde{p}\right)^{T}\left(\tilde{N} u^{\prime}-\tilde{N} u^{\prime \prime}\right) \geqslant 0 .
\end{aligned}
$$

Adding these two inequalities yields

$$
\left(u^{\prime}-u^{\prime \prime}\right)^{T} \tilde{M}^{T} \tilde{N}\left(u^{\prime}-u^{\prime \prime}\right) \leqslant 0 .
$$

Thus

$$
\left(x^{\prime}-x^{\prime \prime}\right)^{T} M^{T} N\left(x^{\prime}-x^{\prime \prime}\right) \leqslant 0
$$

which contradicts the fact that $M^{T} N>0$. Hence, there is only one solution to problem (4).

In the rest of the this section, we consider two special cases of GLVIs in terms of $\mathcal{X}$. As special cases, needless to say, they can be of course solved by using GPNN (17) under appropriate conditions. However, we are concerned with reducing the network complexity based on particular structures of $\mathcal{X}$.

\section{B. GLVIs With Inequality Constraints Only}

We first consider $\mathcal{X}$ in (11) with inequality constraints only; i.e.,

$$
\mathcal{X}=\left\{x \in R^{n} \mid A x \in \Omega_{y}\right\}
$$

where the notations are the same as in (11). According to the arguments in IV-A, a GPNN with its state equation given by

$$
\frac{d u}{d t}=\lambda W\left\{-\check{N} u+P_{\check{U}}((\check{N}-\alpha \check{M}) u+\check{q}-\alpha \check{p})-\check{q}\right\}
$$

can solve the GLVI with $\mathcal{X}$ defined by (18), where

$$
\begin{aligned}
\check{M} & =\left(\begin{array}{cc}
M & -A^{T} \\
0 & I
\end{array}\right) \quad \check{p}=\left(\begin{array}{l}
p \\
0
\end{array}\right) \quad \check{N}=\left(\begin{array}{cc}
N & 0 \\
A N & 0
\end{array}\right) \\
\check{q} & =\left(\begin{array}{c}
q \\
A q
\end{array}\right) \quad \check{U}=R^{n} \times \Omega_{y}
\end{aligned}
$$

$\lambda \in R, \alpha \in R, \lambda>0, \alpha>0$, and $W \in R^{(n+h) \times(n+h)}$. The NN has clearly $n+h$ neurons. In the following, we develop a GPNN with $h$ neurons to solve this problem under an additional condition saying that $M$ is nonsingular.

From Proposition 1, by replacing $\Omega_{x}$ with $R^{n}, \tilde{A}$ with $A$, and $\tilde{\Omega}_{y}$ with $\Omega_{y}$, we have

$$
\left\{\begin{array}{l}
M x^{*}+p-A^{T} y^{*}=0 \\
\left(y^{*}\right)^{T}\left(y-A N x^{*}-A q\right) \geqslant 0 \quad \forall y \in \Omega_{y} .
\end{array}\right.
$$

As $M$ is nonsingular, the previous equality implies $x^{*}=M^{-1} A^{T} y^{*}-M^{-1} p$. Substituting this into the previous inequality gives

$$
\left(y^{*}\right)^{T}\left(y-\hat{N} y^{*}-\hat{q}\right) \geqslant 0 \quad \forall y \in \Omega_{y}
$$


where

$$
\hat{N}=A N M^{-1} A^{T} \quad \hat{q}=-A N M^{-1} p+A q .
$$

Then, the original GLVI is converted into another GLVI with box set $\Omega_{y}$. The following specific GPNN is proposed to solve the problem:

- state equation

$$
\frac{d u}{d t}=\lambda W\left\{-\hat{N} u+P_{\Omega_{y}}((\hat{N}-\alpha I) u+\hat{q})-\hat{q}\right\}
$$

- output equation

$$
v=M^{-1} A^{T} u-M^{-1} p
$$

where $\lambda \in R, \alpha \in R, \lambda>0, \alpha>0$, and $W \in R^{h \times h}$.

All results in Section III can be applied to study the behavior of GPNN (20a) and (20b), by replacing $N, M, W, \mathcal{X}$ with $\hat{N}, I, W, \Omega_{y}$. In the following, we present some results for GPNN (20a) and (20b) that impose no, or simple, restrictions on the constraint parameter $A$.

Theorem 6: Consider GPNN (20a) and (20b) with $W=$ $(\hat{N}+\alpha I)^{T}$ for solving the GLVI with $\mathcal{X}$ defined in (18). Suppose that $M$ is nonsingular. The following are true.

1) If $M^{T} N \geqslant 0$, then $\mathcal{X}^{*}=\left\{v \in R^{n} \mid v=M^{-1} A^{T} u-\right.$ $\left.M^{-1} p, u \in \Omega_{y}^{e}\right\}$, where $\Omega_{y}^{e}$ denotes the set of equilibrium points of (20a), and the state trajectory of the NN is stable in the sense of Lyapunov and globally convergent to an equilibrium point.

2) If $M^{T} N>0$, then the output trajectory of the $\mathrm{NN}$ is globally asymptotically stable at the unique solution $x^{*}$ of the GLVI.

3) If $M^{T} N>0$ and $\operatorname{Rank}(A)=h$, then the state trajectory of the NN is globally exponentially stable.

Proof: If $M^{T} N \geqslant 0$, then $N M^{-1} \geqslant 0$ and $\hat{N}=A N M^{-1} A^{T} \geqslant 0$. Furthermore, if $M^{T} N>0$ and $\operatorname{Rank}(A)=h$, then $\hat{N}>0$. Consequently, parts 1) and 3) of the theorem follow from Proposition 1 and Lemma 5. From parts 1) and 3), the output trajectory of the NN is stable in the Lyapunov sense and globally convergent to the a solution of (4). From Theorem 5, $M^{T} N>0$ implies that the solution of (4) is unique. Hence, the output trajectory is globally asymptotically stable.

\section{GLVIs With Inequality and Equality Constraints}

We next consider the GLVI with $\mathcal{X}$ defined in (11) without any bound constraint, i.e.,

$$
\mathcal{X}=\left\{x \in R^{n} \mid A x \in \Omega_{y}, B x=c\right\}
$$

where the notations are the same as in (11). In this section, we assume that $\operatorname{Rank}(B)=r$ (otherwise, several equalities in $B x=c$ can be removed by some simple technique). To ensure the existence of feasible solutions to the problem, it is necessary that $r \leqslant n$. From Section IV-A, GPNN (17) with $\Omega_{x}$ replaced by $R^{n}$ can solve the GLVI, which has $n+h+r$ neurons. From Section IV-B, by treating $B x=c$ as inequality constraints, a GPNN with $h+r$ neurons may also solve the problem under appropriate conditions. In what follows we show that a GPNN with $h$ neurons may also solve the problem. Let us first present an optimality condition for the GLVI with $\mathcal{X}$ defined in (21).

Proposition 2: $x^{*} \in R^{n}$ is a solution of the GLVI with $\mathcal{X}$ defined by (21) if and only if there exist $y^{*} \in R^{h}$ and $z^{*} \in R^{r}$ such that $A N x^{*}+A q \in \Omega_{y}$ and

$$
\left\{\begin{array}{l}
M x^{*}+p-A^{T} y^{*}-B^{T} z^{*}=0 \\
B N x^{*}+B q-c=0 \\
\left(y^{*}\right)^{T}\left(y-A N x^{*}-A q\right) \geqslant 0 \quad \forall y \in \Omega_{y} .
\end{array}\right.
$$

Proof: The proof is similar to that of Proposition 1, and thus omitted.

Suppose that $M$ is nonsingular. From the first equation in (22), we have

$$
x^{*}=M^{-1} A^{T} y^{*}+M^{-1} B^{T} z^{*}-M^{-1} p .
$$

Substituting it into the second equation in (22) gives

$B N M^{-1} A^{T} y^{*}+B N M^{-1} B^{T} z^{*}-B N M^{-1} p+B q-c=0$.

Assume that $M^{T} N>0$, then $B N M^{-1} B^{T}$ is positive definite because $\operatorname{Rank}(B)=r \leqslant n$; and so is its inverse. It follows that

$$
\begin{aligned}
& z^{*}=-\left(B N M^{-1} B^{T}\right)^{-1} B N M^{-1} A^{T} y^{*} \\
& +\left(B N M^{-1} B^{T}\right)^{-1}\left(B N M^{-1} p-B q+c\right) .
\end{aligned}
$$

Substituting $z^{*}$ into the expression of $x^{*}$ obtained previously gives

$$
x^{*}=D y^{*}+d
$$

where

$$
\begin{aligned}
D= & M^{-1} A^{T}-M^{-1} B^{T}\left(B N M^{-1} B^{T}\right)^{-1} B N M^{-1} A^{T} \\
d= & M^{-1} B^{T}\left(B N M^{-1} B^{T}\right)^{-1} \\
& \times\left(B N M^{-1} p-B q+c\right)-M^{-1} p .
\end{aligned}
$$

Substituting $x^{*}=D y^{*}+d$ into the inequality in (22) results in

$$
\left(y^{*}\right)^{T}\left(y-\bar{N} y^{*}-\bar{q}\right) \geqslant 0 \quad \forall y \in \Omega_{y}
$$

where

$$
\begin{aligned}
\bar{N} & =A N D=A G A^{T} \\
G & =N M^{-1}-N M^{-1} B^{T}\left(B N M^{-1} B^{T}\right)^{-1} B N M^{-1} \\
\bar{q} & =A N d+A q .
\end{aligned}
$$

Based on this equivalent formulation, the following specific GPNN is proposed for solving the GLVI concerned in this section:

- state equation

$$
\frac{d u}{d t}=\lambda W\left\{-\bar{N} u+P_{\Omega_{y}}((\bar{N}-\alpha I) u+\bar{q})-\bar{q}\right\}
$$

- output equation

$$
v=D u+d
$$

where $\lambda \in R, \alpha \in R, \lambda>0, \alpha>0$, and $W \in R^{h \times h}$. 
Lemma 6: Let $H \in R^{n}, P \in R^{m \times n}, H^{T}=H, H>$ $0, \operatorname{Rank}(P)=m$. Then, the matrix

$$
Q=H-H P^{T}\left(P H P^{T}\right)^{-1} P H \geqslant 0 .
$$

Moreover, $\operatorname{Rank}(Q) \leqslant n-m$ and, consequently, $Q$ has at least $m$ zero eigenvalues. In particular, when $m=n, Q=0$.

Proof: Define a matrix

$$
L=\left(\begin{array}{cc}
P H P^{T} & P H \\
H P^{T} & H
\end{array}\right)
$$

which is symmetric. The Schur complement of $P H P^{T}$ in $L$ (see Lemma 4), denoted as $L / P H P^{T}$, is just $Q$. Since $H>$ $0, \operatorname{Rank}(P)=m$, we have $P H P^{T}>0$. Moreover, by noticing that

$$
\begin{aligned}
& \left(\begin{array}{l}
x \\
y
\end{array}\right)^{T}\left(\begin{array}{ll}
H & H \\
H & H
\end{array}\right)\left(\begin{array}{l}
x \\
y
\end{array}\right) \\
& =x^{T} H x+2 x^{T} H y+y^{T} H y \\
& =(x+y)^{T} H(x+y) \geqslant 0 \quad \forall x, y \in R^{n}
\end{aligned}
$$

we have $\left(\begin{array}{ll}H & H \\ H & H\end{array}\right) \geqslant 0$, which indicates that

$$
L=\left(\begin{array}{cc}
P^{T} & 0 \\
0 & I
\end{array}\right)^{T}\left(\begin{array}{cc}
H & H \\
H & H
\end{array}\right)\left(\begin{array}{cc}
P^{T} & 0 \\
0 & I
\end{array}\right) \geqslant 0 .
$$

By Lemma 4, we conclude that $Q \geqslant 0$. In addition, it is obvious that $P Q=0$. If $m=n$, then $Q=0$ because $\operatorname{Rank}(P)=$ $m$. Suppose that $m<n$. Denote $p_{i}^{T}$ the $i$ th row of $P$ with $i=1, \ldots, m$, and $q_{j}$ the $j$ th column of $Q$ with $j=1, \ldots, n$. Then, $\Upsilon=\operatorname{span}\left\{p_{1}, \ldots, p_{m}\right\}$ is a subspace of $R^{n}$. The dimensionality of its orthogonal complement, denoted by $\Upsilon^{\perp}$, is $(n-m)$. Clearly, $q_{j} \in \Upsilon^{\perp}, \forall j=1, \ldots, n$. Therefore, among these $q_{j}$ 's, at most $(n-m)$ are linearly independent. Then, $\operatorname{Rank}(Q) \leqslant n-m$. Because $Q=Q^{T} \geqslant 0$, it can be decomposed as $Q=T \Lambda T^{T}$, where $T$ is an orthogonal matrix and $\Lambda=\operatorname{diag}\left(\lambda_{1}, \ldots, \lambda_{n}\right)$ with $\lambda_{i} \geqslant 0$ being the eigenvalues of $Q$. Then, $\operatorname{Rank}(Q)=\operatorname{Rank}(\Lambda) \leqslant n-m$. It follows that at least $m$ eigenvalues of $Q$ are equal to zero. In particular, if $m=n$, $Q=0$.

Lemma 6 indicates that $\bar{N}$ in (23) is positive semidefinite. Then, the following results follow from Lemma 5.

Theorem 7: Consider GPNN (24a) and (24b) with $W=$ $(\bar{N}+\alpha I)^{T}$ for solving the GLVI with $\mathcal{X}$ defined in (21). If $\operatorname{Rank}(B)=r, M$ is nonsingular and $M^{T} N$ is symmetric and positive definite, then the following hold.

1) $\mathcal{X}^{*}=\left\{v \in R^{n} \mid v=D u+d, u \in \Omega_{y}^{e}\right\}$, where $\Omega_{y}^{e}$ denotes the set of equilibrium points of (24a).

2) The state trajectory of the NN is stable in the sense of Lyapunov and globally convergent to an equilibrium point.

Remark 4: Note that the conditions $M^{T} N>0$ and $M^{T} N=$ $N^{T} M$ in Theorem 7 ensure $\bar{N} \geqslant 0$ and $\bar{N}^{T}=\bar{N}$ (see Lemma 6). In view of Lemma 5, Theorem 7 still holds if $\bar{N}$ is positive semidefinite but asymmetric. The reason for imposing the symmetric condition on $M^{T} N$ and, hence, on $\bar{N}$ is that, at current stage, we find no rigorous evidence showing that $\bar{N} \geqslant 0$ when $M^{T} N$ is asymmetric and positive definite, though numerical experiments agree with this expectation (refer to Example 3 in Section V). This could be an open problem.
Remark 5: If we could assume $G>0$ and $\operatorname{Rank}(A)=h$ in (24a) and (24b), then $\bar{N}>0$ because $\bar{N}=A G A^{T}$. Consequently, we could conclude the global exponential stability of GPNN (24a) and (24b) from Lemma 5. However, Lemma 6 smashes this conjecture, which says whenever the equality constraints $B x=c$ are present, $G$ cannot be positive definite but positive semidefinite only.

A comparison of the three GPNN models in (17), (20a), (20b), (24a), and (24b) shows that, to guarantee the same convergence properties, the required conditions are in an order from weak to strong (see Theorems 5-7), though their structures are getting simpler and simpler. For example, GPNN (20a) and (20b) requires $M$ to be nonsingular while GPNN (17) does not. For another example, to ensure the global convergence, both GPNNs in (17) and (20) require the positive semidefiniteness of $M^{T} N$ only, while GPNN (24a) and (24b) requires the positive definiteness of this matrix. It should be regarded as weakness of the latter two NNs. Nevertheless, according to Remark 4, some additional conditions required by GPNN (24a) and (24b) might not be necessary. Moreover, this comparison is based on Theorems 5-7 which are derived from Lemma 5. If the three GPNNs are compared based on other stability results presented in Section III, no obvious weakness or strength of any GPNN can be observed in general (see Example 4 in Section V).

\section{NUMERICAL EXAMPLES}

Example 1: First, consider a GLVI with bound constraints only. Let the parameters in (4) be defined as

$$
\begin{aligned}
M & =\left(\begin{array}{ccc}
5 & -6 & 1 \\
10 & 4 & 6 \\
-5 & -3 & 4
\end{array}\right) \quad N=\left(\begin{array}{ccc}
9 & -13 & 3 \\
19 & 10 & 17 \\
-11 & -5 & 9
\end{array}\right) \\
p & =\left(\begin{array}{c}
-1 \\
2 \\
5
\end{array}\right) \quad q=\left(\begin{array}{l}
0 \\
2 \\
0
\end{array}\right)
\end{aligned}
$$

and $\mathcal{X}=\left\{x \in R^{3} \mid(-4,0,-4)^{T} \leqslant x \leqslant(2,4,2)^{T}\right\}$. Since $M^{T} N>0$ and $N+\alpha M$ is not symmetric for any $\alpha>0$, according to Lemma 5, GPNN (6) with $W=(N+\alpha M)^{T}$ can solve the problem, but it is not sure whether GPNN (6) with $W=I$ can do it. Let $P=\operatorname{diag}(1,0.5,0.8)$ and $\alpha=2$; then, it can be verified that $Q=P M+M^{T} P>0$ and

$$
2\|P\|\|N-\alpha M\|=5.94<6.31=\alpha \theta_{\min }(Q) .
$$

According to Theorem 2, GPNN (6) with $W=I$ and $\alpha=2$ can also solve the problem. Simulation results show that GPNN (6) converges to the unique solution of the problem $(0.1728,-0.0678,-0.2709)^{T}$ from any initial point in $R^{3}$. For example, Fig. 1(a) demonstrates the transient behavior of such a GPNN (6) with $\lambda=1$ from a random initial point, and Fig. 1(b) shows the evolution of the error $\left\|x(t)-x^{*}\right\|$. It is seen that the convergence rate of the trajectories $x(t)$ is exponential.

Example 2: Consider a GLVI with

$$
M=I \quad N=\left(\begin{array}{ccc}
9 & -6 & 4 \\
-7 & 6 & 8 \\
-5 & 2 & 3
\end{array}\right) \quad p=\left(\begin{array}{l}
2 \\
2 \\
5
\end{array}\right) \quad r q=\left(\begin{array}{l}
0 \\
0 \\
0
\end{array}\right)
$$




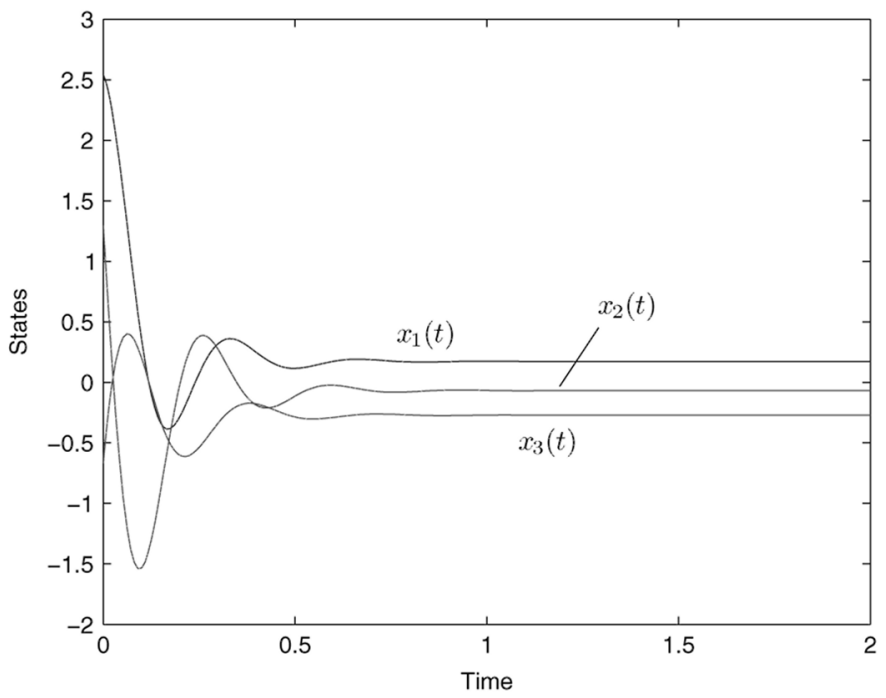

(a)

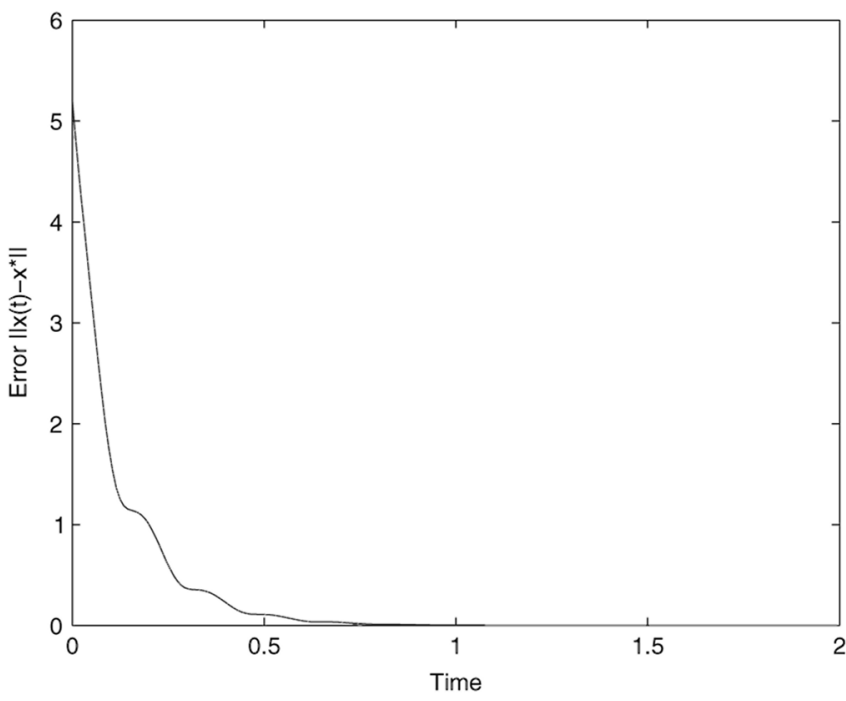

(b)

Fig. 1. Convergence behavior of GPNN (6) with $\lambda=1, W=I$, and $\alpha=2$ from a random initial point in Example 1. (a) State trajectory. (b) Error.

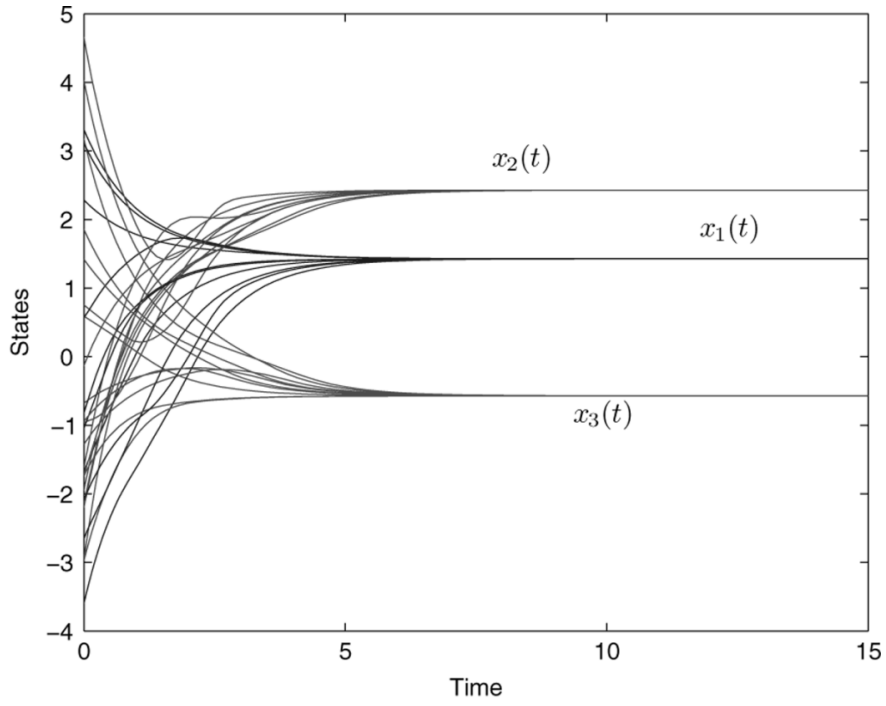

Fig. 2. State trajectories of GPNN (6) with $\lambda=1, W=N^{-1}$, and $\alpha=1$ from ten random initial points in Example 2.

and $\mathcal{X}$ as the same as in Example 1. Since $N$ is not positive semidefinite, if $W=(N+\alpha M)^{T}$ is used in GPNN (6), one cannot ascertain the global convergence of the $\mathrm{NN}$ according to Lemma 5. However, by noticing that $N^{-1} \in L D S$ since $\Gamma N^{-1}>0$ where $\Gamma=\operatorname{diag}(1,2,5)$, we can let $W=N^{-1}$ in GPNN (6) and ensure the global exponential stability of the resulting NN according to Theorem 3. Fig. 2 shows the trajectories of such a GPNN (6) with $\lambda=\alpha=1$ from ten random initial points. It can be seen that all trajectories converge to the unique solution of the GLVI $(1.4283,2.4284,-0.5712)^{T}$.

Example 3: Consider a GLVI with a general polyhedron defined in (11). Let

$$
\begin{aligned}
M & =\left(\begin{array}{ccc}
1 & -1 & -1 \\
-1 & 1 & 0 \\
0 & 1 & -1
\end{array}\right) \quad N=\left(\begin{array}{ccc}
1 & -1 & -1 \\
0 & -1 & 0 \\
0 & 3 & -1
\end{array}\right) \\
p & =\left(\begin{array}{c}
-1 \\
-1 \\
2
\end{array}\right) \quad q=\left(\begin{array}{l}
0 \\
2 \\
0
\end{array}\right) .
\end{aligned}
$$

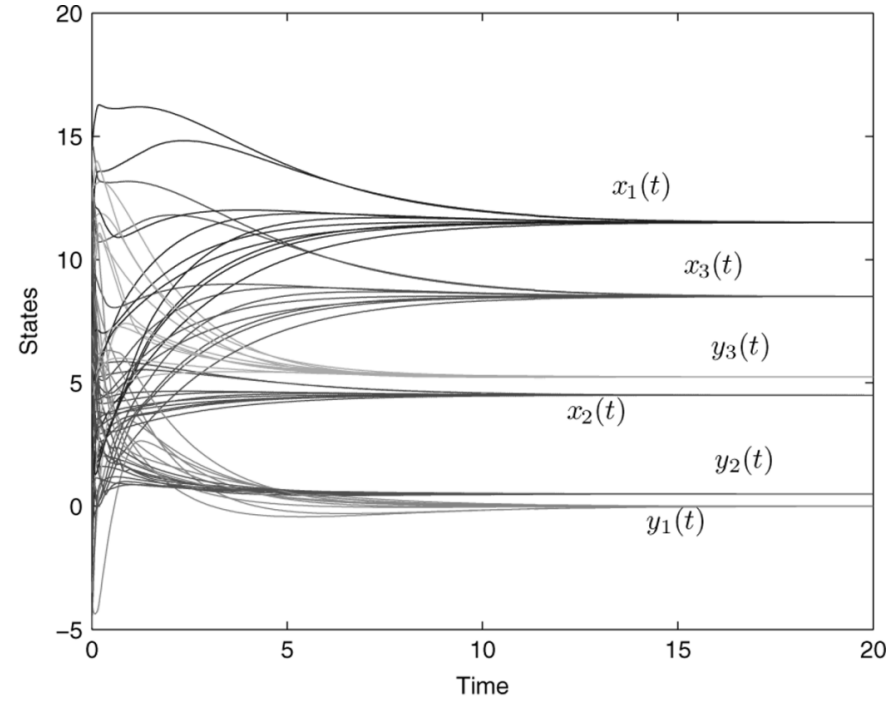

Fig. 3. State trajectories of GPNN (17) with $\lambda=1, W=(\tilde{N}+\alpha \tilde{M})^{T}$, and $\alpha=1$ from ten initial points in Example 3 where bound constraints are present.

We first consider the case when all constraints in (11) are present, where

$$
A=\left(\begin{array}{ccc}
1 & 1 & 0 \\
-5 & 5 & -1
\end{array}\right) \quad B=\left(\begin{array}{lll}
0 & -2 & 1
\end{array}\right) \quad c=10
$$

and $\Omega_{x}=\left\{x \in R^{3} \mid-5 \leqslant x \leqslant 5\right\}, \Omega_{y}=\left\{y \in R^{2} \mid-10 \leqslant\right.$ $y \leqslant 10\}$. It can be verified that $M^{T} N>0$. GPNN (17) with $W=(N+\alpha \tilde{M})^{T}$ can be used to solve the problem according to Theorem 5. Simulation results show that from any initial point this NN globally converges to the unique equilibrium point $u^{*}=$ $(11.5000,4.5000,8.5000,-0.0000,0.5000,5.2500)^{T}$. That is, the unique solution of the GLVI is $x^{*}=(11.5,4.5,8.5)^{T}$. Fig. 3 displays the transient behavior of the NN with $\lambda=\alpha=1$ and ten different initial points.

Next, we let $\Omega_{x}=R^{3}$ in (11) and solve the GLVI without bound constraints by using GPNN (17) with $W=(\tilde{N}+\alpha \tilde{M})^{T}$. The solution obtained is $x^{*}=(5.7868,3.4420,3.2100)^{T}$. Since the bound constraints in $\mathcal{X}$ is removed, GPNN (24a) and (24b) 


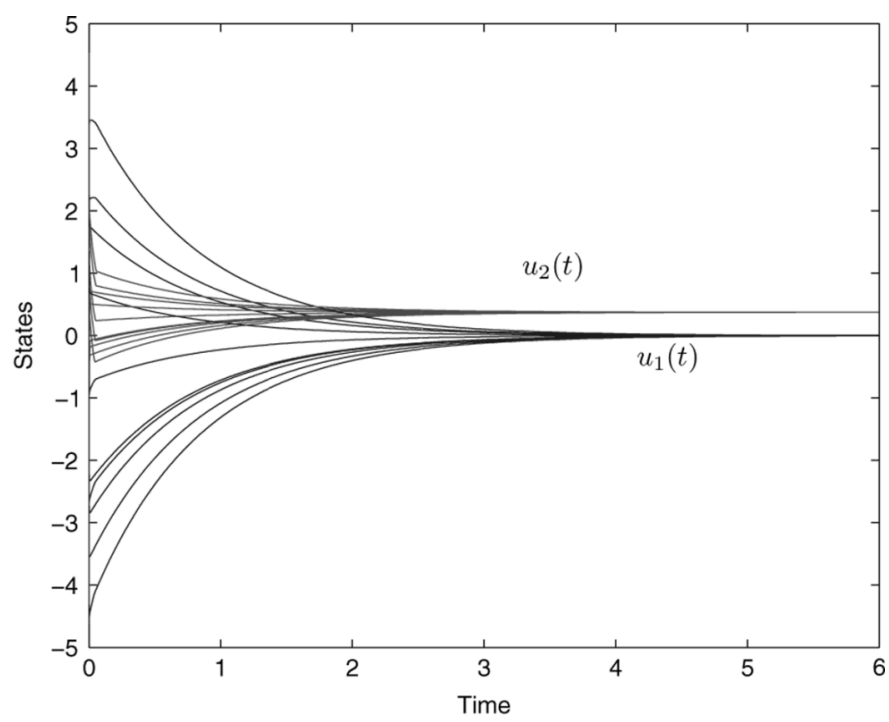

Fig. 4. State trajectories of GPNN (24a) and (24b) with $\lambda=1, W=(\bar{N}+$ $\alpha I)^{T}$, and $\alpha=1$ from ten initial points in Example 3 where bound constraints are absent.

may also solve the problem. Fig. 4 shows the state trajectories of GPNN (24a) and (24b) with $\lambda=\alpha=1$ and $W=$ $(\bar{N}+\alpha I)^{T}$ from ten random initial points in $R^{3}$. It is seen that, though $M^{T} N$ is asymmetric, all trajectories converge to $u^{*}=(0.0000,0.3730)^{T}$, which corresponds to the solution of the GLVI $x^{*}=(5.7868,3.4420,3.2100)^{T}$. This phenomenon echoes Remark 4.

Example 4: Let us solve a GLVI in (4) with inequality constraints only, which is determined by

$$
\begin{aligned}
M & =\left(\begin{array}{cc}
-1 & 2 \\
0 & 2
\end{array}\right) \quad N=\left(\begin{array}{ll}
1 & 1 \\
3 & 1
\end{array}\right) \quad p=\left(\begin{array}{l}
10 \\
20
\end{array}\right) \\
q & =\left(\begin{array}{l}
-10 \\
-20
\end{array}\right)
\end{aligned}
$$

and $\mathcal{X}$ is defined in (18) with

$$
A=\left(\begin{array}{cc}
-1 & 1 \\
2 & 3
\end{array}\right)
$$

and $\Omega_{y}=\left\{y \in R^{2} \mid-10 \leqslant y \leqslant 0\right\}$. For solving the problem, there are two possible choices of GPNNs, i.e., (17) and (20a)-(20b). If the former is used, Theorem 5 cannot be applied since $M^{T} N$ is neither positive definite nor positive semidefinite. Moreover, in this case, many stability results for (17) tailored from those for (6) discussed in Section III (e.g., Lemma 5, Theorems 3 and 4) by replacing $M, N, p, q$, and so on with $\tilde{M}, \tilde{N}, \tilde{q}, \tilde{q}$, and so on, cannot be applied either, since it is easily seen that

$$
\tilde{N}=\left(\begin{array}{cc}
N & 0 \\
A N & 0
\end{array}\right)
$$

is singular and

$$
\tilde{M}^{T} \tilde{N}=\left(\begin{array}{cc}
M^{T} N & 0 \\
0 & 0
\end{array}\right)
$$

is not positive (semi-)definite. However, if the latter is used, simple calculations yield

$$
\hat{N}=A N M^{-1} A^{T}=\left(\begin{array}{cc}
4 & 2 \\
24 & 18.5
\end{array}\right)
$$

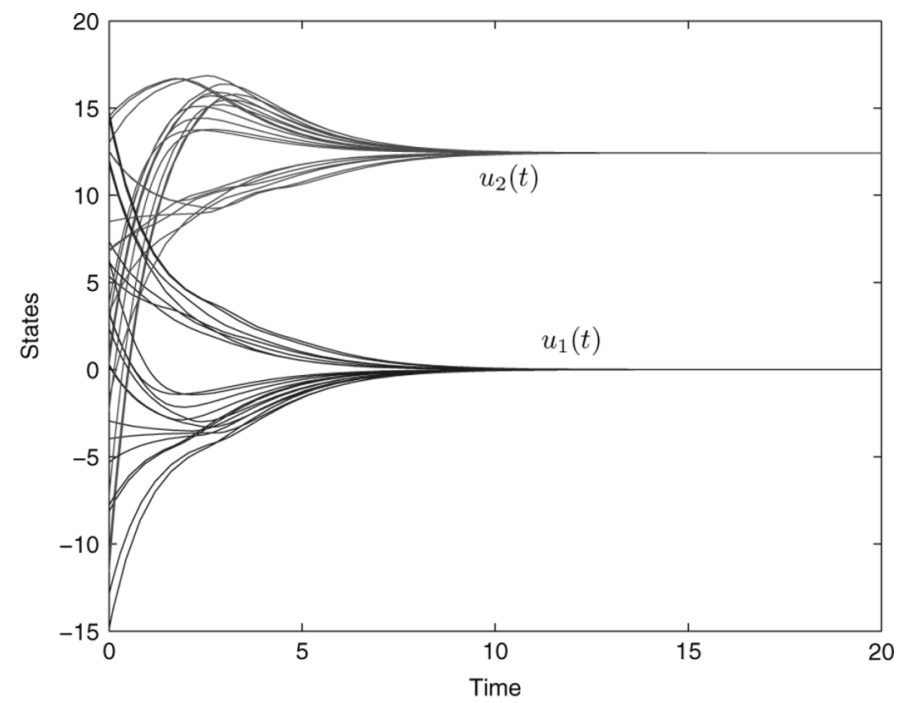

Fig. 5. State trajectories of GPNN (20a) and (20b) with $\lambda=1, W=\hat{N}^{-1}$, and $\alpha=1$ from 20 initial points in Example 4.

which is nonsingular, and

$$
I-\alpha \hat{N}^{-1}=\left(\begin{array}{ll}
0.26 & 0.08 \\
0.98 & 0.84
\end{array}\right)
$$

when $\alpha=1$. Note that there exist $\varrho_{1}=10$ and $\varrho_{1}=70$ such that $0.26 \varrho_{1}+0.08 \varrho_{2}<\varrho_{1}$ and $0.98 \varrho_{1}+0.84 \varrho_{2}<\varrho_{2}$. According to Theorem 4, GPNN (20a) and (20b) with $W=$ $\hat{N}^{-1}$ is globally exponentially stable at the unique solution of the previous GLVI. Simulation results verify this fact. Fig. 5 illustrates the state trajectories of GPNN (20a) and (20b) with $\lambda=\alpha=1$ from 20 random initial points, from which it is seen that all trajectories converge to a unique point $u^{*}$. By using the output (20b), the solution of the GLVI is calculated as $x^{*}=$ $v^{*}=(2.4324,8.6486)^{T}$.

Example 5: In this example, we would like to show the numerical performance of the NNs based on numerical solvers of ordinary differential equations (ODE), because, in general, the dynamic behavior of recurrent NNs can be simulated via ODE solvers, as seen in the preceding examples. We do this by comparing the performance of an ODE solver for NN simulation with two iterative numerical algorithms. However, most existing iterative numerical algorithms are for solving LVIs and NVIs and there are few for solving GLVI (4), especially with a general polyhedral set $\mathcal{X}$ defined in (11); so, we will consider to solve LVIs (1) (i.e., GLVIs (4) with $N=I$ and $q=0$ ). For simplicity, let the constrained set be defined in (11) with $\underline{x}=0$ and $\bar{x}=\infty$. For solving such type of LVIs, [4] provides an interesting equivalent constrained optimization formulation

$$
\begin{aligned}
& \min f(x, y, z, u, v) \\
& =\left\|M x+p+\left(A^{T},-A^{T}\right) y+B^{T} u-v\right\|^{2} \\
& +\rho_{1}\left\|z+\left(A^{T},-A^{T}\right)^{T} x+\left(-\bar{y}^{T}, \underline{y}^{T}\right)^{T}\right\|^{2} \\
& +\rho_{2}\|B x-c\|^{2}+\rho_{3}\left(\left(y^{T} z\right)^{p}+\left(x^{T} v\right)^{p}\right), \\
& \text { s.t. } x \geq 0, \quad y \geq 0 ; \quad z \geq 0 ; \quad v \geq 0
\end{aligned}
$$

where $\rho_{1}, \rho_{2}, \rho_{3}$, and $p$ are positive constants. From [4], $x^{*}$ solves (1) if and only if there exist $y^{*}, z^{*} \in R_{+}^{2 h}, u^{*} \in R^{r}$, 
TABLE I

NUMERICAL COMPARISON RESULTS

\begin{tabular}{|c|c|c|c|c|c|c|c|c|}
\hline \multirow{2}{*}{ Problem Size } & \multicolumn{2}{|c|}{ GPNN1 } & \multicolumn{2}{|c|}{ GPNN2 } & \multicolumn{2}{|c|}{ SPG1 } & \multicolumn{2}{|c|}{ SPG2 } \\
\hline & Error & CPU Time & Error & CPU Time & Error & CPU Time & Error & CPU Time \\
\hline & $10^{-5}$ & 6.3 & $2.0 \times 10^{-4}$ & 600 & $10^{-5}$ & 245.9 & $10^{-5}$ & 236.8 \\
\hline$n=5$ & $10^{-5}$ & 10.9 & $3.8 \times 10^{-3}$ & 600 & $10^{-5}$ & 432.5 & $10^{-5}$ & 439.3 \\
\hline$h=3$ & $10^{-5}$ & 20.1 & $2.6 \times 10^{-3}$ & 600 & $3.9 \times 10^{-4}$ & 600.0 & $10^{-5}$ & 406.9 \\
\hline \multirow[t]{2}{*}{$r=1$} & $10^{-5}$ & 2.4 & $1.1 \times 10^{-3}$ & 600 & $10^{-5}$ & 112.7 & $10^{-5}$ & 112.7 \\
\hline & $10^{-5}$ & 6.2 & $1.0 \times 10^{-3}$ & 600 & $10^{-5}$ & 32.7 & $10^{-5}$ & 32.5 \\
\hline & $10^{-5}$ & 11.2 & $1.8 \times 10^{-3}$ & 600 & $10^{-5}$ & 57.9 & $10^{-5}$ & 52.0 \\
\hline$n=10$ & $10^{-5}$ & 23.8 & $3.4 \times 10^{-3}$ & 600 & $10^{-5}$ & 116.6 & $10^{-5}$ & 144.2 \\
\hline$h=$ & $10^{-5}$ & 11.6 & $1.0 \times 10^{-3}$ & 600 & $10^{-5}$ & 59.8 & $10^{-5}$ & 52.4 \\
\hline \multirow[t]{2}{*}{$r=2$} & $10^{-5}$ & 30.0 & $2.1 \times 10^{-4}$ & 600 & $10^{-5}$ & 247.8 & $10^{-5}$ & 172.3 \\
\hline & $10^{-5}$ & 30.2 & $10^{-5}$ & 0.8 & $10^{-5}$ & 152.6 & $10^{-5}$ & 51.6 \\
\hline & $6.3 \times 10^{-5}$ & 1200 & $5.6 \times 10^{-5}$ & 1200 & $2.8 \times 10^{-2}$ & 1200 & $2.7 \times 10^{-2}$ & 1200 \\
\hline$n=30$ & $1.5 \times 10^{-3}$ & 1200 & $2.4 \times 10^{-3}$ & 1200 & $4.6 \times 10^{-3}$ & 1200 & $4.5 \times 10^{-3}$ & 1200 \\
\hline$h=10$ & $4.9 \times 10^{-3}$ & 1200 & $1.5 \times 10^{-3}$ & 1200 & $7.1 \times 10^{-3}$ & 1200 & $5.0 \times 10^{-3}$ & 1200 \\
\hline \multirow{2}{*}{$r=10$} & $10^{-5}$ & 999.1 & $1.3 \times 10^{-4}$ & 1200 & $6.1 \times 10^{-3}$ & 1200 & $2.1 \times 10^{-2}$ & 1200 \\
\hline & $2.4 \times 10^{-4}$ & 1200 & $2.3 \times 10^{-3}$ & 1200 & $1.4 \times 10^{-2}$ & 1200 & $1.7 \times 10^{-3}$ & 1200 \\
\hline \multirow{5}{*}{$\begin{array}{l}n=50 \\
h=20 \\
r=10\end{array}$} & $7.1 \times 10^{-4}$ & 1800 & $5.8 \times 10^{-5}$ & 1800 & $7.1 \times 10^{-3}$ & 1800 & $2.7 \times 10^{-2}$ & 1800 \\
\hline & $1.8 \times 10^{-3}$ & 1800 & $2.4 \times 10^{-5}$ & 1800 & $4.2 \times 10^{-2}$ & 1800 & $1.1 \times 10^{-2}$ & 1800 \\
\hline & $9.8 \times 10^{-5}$ & 1800 & $2.2 \times 10^{-3}$ & 1800 & $4.9 \times 10^{-2}$ & 1800 & $1.1 \times 10^{-2}$ & 1800 \\
\hline & $1.0 \times 10^{-4}$ & 1800 & $10^{-5}$ & 5.5 & $3.7 \times 10^{-2}$ & 1800 & $4.4 \times 10^{-3}$ & 1800 \\
\hline & $3.0 \times 10^{-4}$ & 1800 & $4.9 \times 10^{-4}$ & 1800 & $1.1 \times 10^{-2}$ & 1800 & $4.1 \times 10^{-2}$ & 1800 \\
\hline
\end{tabular}

and $v^{*} \in R_{+}^{n}$ such that $w^{*}=\left(x^{*}, y^{*}, z^{*}, u^{*}, v^{*}\right)$ is a global minimizer of (25) and $f\left(w^{*}\right)=0$. Moreover, if $p>1, M>0$, and $\left(x^{*}, y^{*}, z^{*}, u^{*}, v^{*}\right)$ constitute a constrained stationary point of (25), then $x^{*}$ is a solution of LVI (1). Hence, one can solve this LVI by solving (25). Here, we adopt two numerical algorithms, SPG1 and SPG2, in [25] for solving (25).

The test problems are generated as follows. First, two $n \times n$ matrices $G_{0}$ and $G_{1}$ with random entries between -3 and 3 are generated. Let $G_{3}=G_{0} G_{0}^{T}$ and $G_{4}=G_{1} G_{1}^{T}$. Then, define $G_{5}=\left(G_{5}\right)_{i j} \in R^{n \times n}$ an upper triangular matrix such that $\left(G_{5}\right)_{i i}=\left(G_{4}\right)_{i i} / 2, \forall i=1, \ldots, n$ and $\left(G_{5}\right)_{i j}=\left(G_{4}\right)_{i j}, \forall i<$ $j$. Finally, let $M=G_{3}+G_{5}$. This procedure is taken from [3], which ensures $M \geq 0$ but not necessarily symmetric. The other parameters in (1) and (11) are generated as follows. All entries of $p$ are random numbers in [0,20], all entries of $A$ and $B$ are random numbers in $[-10,10]$, and all entries of $c$ are random numbers in $[-20,20] . \underline{y}=-(50, \ldots, 50)^{T}, \bar{y}=$ $(50, \ldots, 50)^{T}$. Three sets of problems are generated with different combinations of $n, h$, and $r$, and in each set five problems are generated (see Table I).

The NN (17) is simulated to solve the problems based on the ODE solver "ode45" in MATLAB 7.0. For convenience, hereafter, the NN with $W=(\tilde{N}+\alpha \tilde{M})^{T}$ is called GPNN1 and the NN with $W=I$ is called GPNN2. In all simulations, both $\lambda$ and $\alpha$ are set to 1 . The initial points are always randomly generated in $[-10,10]^{n+h+r}$. The following stopping criteria are adopted:

- $\left\|-\tilde{N} u+P_{\tilde{U}}((\tilde{N}-\alpha \tilde{M}) u+\tilde{q}-\alpha \tilde{p})-\tilde{q}\right\| /(n+h+r) \geq$ $10^{-5}$

- CPU time is equal to or greater than $600 \mathrm{~s}$ for the first two sets of problems, $1200 \mathrm{~s}$ for the third set of problems, and $1800 \mathrm{~s}$ for the fourth set of problems.

Note that $\left\|-\tilde{N} u+P_{\tilde{U}}((\tilde{N}-\alpha \tilde{M}) u+\tilde{q}-\alpha \tilde{p})-\tilde{q}\right\|=0$ if and only if an exact solution is achieved. Thus, the first aforementioned stopping criterion measures the normalized error of every dimension of the state variable $u$. In (25), set $\rho_{1}=\rho_{2}=\rho_{3}=$ $p=2$ as suggested in [4]. The two algorithms SPG1 and SPG2 are coded in MATLAB 7.0, and the algorithmic parameters are adopted as recommended in [25]. The initial points for the algorithms are also randomly generated in $[-10,10]^{2 n+4 h+r}$. The following stopping criteria are adopted:

- $\left\|-w+P_{\Omega}(w-\nabla f(w))\right\| /(2 n+4 h+r) \geq 10^{-5}$, where $w=(x, y, z, u, v), \Omega=R_{+}^{n} \times R_{+}^{2 h} \times R_{+}^{2 h} \times R^{r} \times R_{+}^{n}$, and $\nabla f$ denotes the gradient of $f(w)$;

- CPU time is equal to or greater than $600 \mathrm{~s}$ for the first two sets of problems, $1200 \mathrm{~s}$ for the third set of problems, and $1800 \mathrm{~s}$ for the fourth set of problems.

Note that $\left\|-w+P_{\Omega}(w-\nabla f(w))\right\|=0$ if and only if an exact solution is achieved. Thus, the first aforementioned stopping criterion measures the normalized error of every dimension of the variable $w$. These criteria make the comparison fair to some extend. Table I lists detailed results of the four algorithms. In the table, for GPNN1 and GPNN2, "Error" refers to $\|-\tilde{N} u+$ $P_{\tilde{U}}((\tilde{N}-\alpha \tilde{M}) u+\tilde{q}-\alpha \tilde{p})-\tilde{q} \| /(n+h+r)$ and for SPG1 and SPG2, "Error" refers to $\left\|-w+P_{\Omega}(w-\nabla f(w))\right\| /(2 n+4 h+r)$. "CPU Time" refers to the execution time (in seconds) on a PC with an Intel Pentium IV 3.2-GHz CPU and a 1024-MB physical memory.

From Table I, it is seen that GPNN1 outperforms SPG1 and SPG2 in terms of convergence time for solving small-sized problems; i.e., the first and second set of problems. For solving the third and fourth set of problems, GPNN1 is at least competitive to SPG1 and SPG2. Another observation from the table is that, though not theoretically founded, GPNN2 converges to the solution of every test problem also. This is not a strange phenomenon by recalling that we have provided some sufficient conditions on the convergence of GPNN2 in Section III, and many other sufficient conditions may exist but have not been revealed yet. However, the speed is lower than GPNN1 for most problems with several exceptions. It is interesting to note that for solving the last problem in the second set and the fourth problem in the fourth set, the convergence time of GPNN2 are surprisingly short. 


\section{CONCLUDING REMARKS}

In this paper, we present some new results on the GPNN for solving GLVIs. In view that the NN is limited to handle the box- or sphere-type constrained GLVIs, we develop a new design methodology for the GPNN to solve GLVIs with general polyhedral constraints. By utilizing optimization techniques, the problems are transformed into new GLVIs with box-type constraints, which then can be solved by using specifically designed GPNNs. In addition, much effort has been devoted to minimize the number of neurons of the designed GPNNs for GLVIs with specific types of constraints in order to reduce the network complexity. Though all of the stability results of GPNN are applicable to the specific GPNNs, we further present some new results based on their particular structures. Finally, several numerical examples are presented to illustrate the performances of the GPNNs. It is interesting to note that in terms of numerical simulation, the $\mathrm{NN}$ is competitive to two iterative algorithms, which reveals the great potential of this method.

\section{REFERENCES}

[1] P. T. Harker and J. S. Pang, "Finite-dimensional variational inequality and nonlinear complementarity problems: A survey of theory, algorithms and applications," Math. Program., vol. 48, pp. 161-220, 1990.

[2] F. Facchinei and J. S. Pang, Finite-Dimensional Variational Inequalities and Complementarity Problems. New York: Springer-Verlag, 2003, vol. I, II.

[3] A. Friedlander, J. M. Martinez, and S. A. Santos, "A new strategy for solving variational inequalities in bounded polytopes," Numer. Functional Anal. Optim., vol. 16, pp. 653-668, 1995.

[4] R. Andreani, A. Friedlander, and J. M. Martinez, "Solution of finitedimensional variational inequalities using smooth optimization with simple bounds," J. Optim. Theory Appl., vol. 94, pp. 635-657, 1997.

[5] J. M. Peng and M. Fukushima, "A hybrid Newton method for solving the variational inequality problem via the D-gap function," Math. Program., ser. A, vol. 86, pp. 367-386, 1999.

[6] R. Andreani and J. M. Martinez, "Reformulation of variational inequalities on a simplex and compactification of complementarity problems," SIAM J. Optim., vol. 10, pp. 878-895, 2000.

[7] Y. Xia and J. Wang, "A recurrent neural network for solving linear projection equations," Neural Netw., vol. 13, no. 3, pp. 337-350, 2000.

[8] X. Liang and J. Si, "Global exponential stability of neural networks with globally Lipschitz continuous activations and its application to linear variational inequality problem," IEEE Trans. Neural Netw., vol. 12, no. 2, pp. 349-359, Mar. 2001.

[9] Y. Xia, H. Leung, and J. Wang, "A projection neural network and its application to constrained optimization problems," IEEE Trans. Circuits Syst. I, Fundam. Theory Appl., vol. 49, no. 4, pp. 447-458, Apr. 2002.

[10] Y. Xia, G. Feng, and J. Wang, "A recurrent neural network with exponential convergence for solving convex quadratic program and related linear piecewise equations," Neural Netw., vol. 17, pp. 1003-1015, 2004.

[11] Y. Xia, "An extended projection neural network for constrained optimization," Neural Comput., vol. 16, pp. 863-883, 2004.

[12] X. B. Gao, L. Z. Liao, and L. Q. Qi, "A novel neural network for variational inequalities with linear and nonlinear constraints," IEEE Trans. Neural Netw., vol. 16, no. 6, pp. 1305-1317, Nov. 2005.

[13] Y. Xia and J. Wang, "Global asymptotic and exponential stability of a dynamic neural system with asymmetric connection weights," IEEE Trans. Autom. Control, vol. 46, no. 4, pp. 635-638, Apr. 2001.
[14] S. Hu and J. Wang, "Global stability of a class of continuous-time recurrent neural networks," IEEE Trans. Circuits Syst. I, Fundam. Theory Appl., vol. 49, no. 9, pp. 1334-1347, Sep. 2002.

[15] B. He, "Solution and applications of a class of general linear variational inequalties," Sci. China Ser. A-Math., vol. 39, pp. 397-404, 1996.

[16] X. B. Gao, "A neural network for a class of extended linear variational inequalities," Chinese J. Electron., vol. 10, pp. 471-475, 2001.

[17] Y. Xia and J. Wang, "A general projection neural network for solving monotone variational inequalities and related optimization problems," IEEE Trans. Neural Netw., vol. 15, no. 2, pp. 318-328, Mar. 2004.

[18] M. Forti and A. Tesi, "New conditions for global stability of neural networks with application to linear and quadratic programming problems," IEEE Trans. Circuits Syst. I, Fundam. Theory Appl., vol. 42, no. 7, pp. 354-366, Jul. 1995.

[19] S. Arik, "A note on the global stability of dynamical neural networks," IEEE Trans. Circuits Syst. I, Fundam. Theory Appl., vol. 49, no. 4, pp. 502-504, Apr. 2002.

[20] J. S. Pang and J. C. Yao, "On a generalization of a normal map and equations," SIAM J. Control Optim., vol. 33, pp. 168-184, 1995.

[21] R. A. Horn and F. Zhang, "Basic properties of the Schur complement," in The Schur Complement and Its Applications, F. Zhang, Ed. New York: Springer-Verlag, 2005.

[22] Z. Zeng, J. Wang, and X. Liao, "Global exponential stability of a general class of recurrent neural networks with time-varying delays," IEEE Trans. Circuits Syst. I, Fundam. Theory Appl., vol. 50, no. 10, pp. 1353-1358, Oct. 2003.

[23] M. S. Bazaraa, H. D. Sherali, and C. M. Shetty, Nonlinear Programming: Theory and Algorithms. New York: Wiley, 1993.

[24] D. Kinderlehrer and G. Stampacchia, An Introduction to Variational Inequalities and Their Applications. New York: Academic, 1980.

[25] E. G. Birgin, J. M. Martinez, and M. Raydan, "Nonmonotone spectral projected gradient methods on convex sets," SIAM J. Optim., vol. 10, no. 4, pp. 1196-1211, 2000.

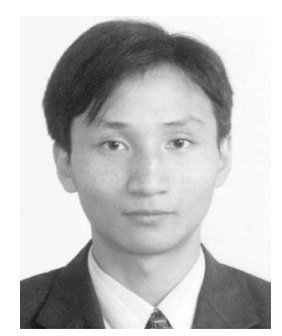

Xiaolin Hu (S'01) received the B.E. and M.E. degrees in automotive engineering from Wuhan University of Technology, Hubei, China, and the Ph.D. degree in mechanical and automation engineering from The Chinese University of Hong Kong, Hong Kong, China, in 2001, 2004, and 2007, respectively.

His current research interests include theories and applications of neural networks and evolutionary computation.

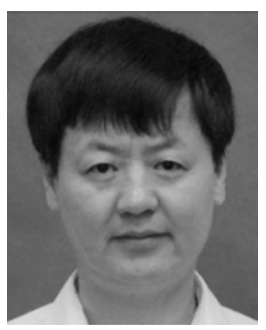

Jun Wang (S'89-M'90-SM'93-F'07) received the B.S. degree in electrical engineering and the M.S. degree in systems engineering from Dalian University of Technology, Dalian, China, and the Ph.D. degree in systems engineering from Case Western Reserve University, Cleveland, OH, in 1982, 1985, and 1991, respectively.

He is a Professor at the Department of Mechanical and Automation Engineering, The Chinese University of Hong Kong, Hong Kong, China. He was an Associate Professor at the University of North Dakota, Grand Forks, until 1995. His current research interests include neural networks and their engineering applications.

Dr. Wang is an Associate Editor of the IEEE TRANSACTIONS ON NeURAL NeTWORKS and the IEEE TRANSACTIONS ON SYSTEMS, MAN, AND CYBERNETICS-PART B: CYBERNETICS, and a member of Editorial Advisory Board of the International Journal of Neural Systems. He is a Past President of the Asia Pacific Neural Network Assembly. 